\title{
The Effect of Intravenous Insulin Infusion on Kidney Function in Insulin-Dependent Diabetes Mellitus
}

\author{
J. Sandahl Christiansen, M. Frandsen, and H.-H. Parving \\ Steno Memorial Hospital, Gentofte, and Departments of Clinical Physiology and Medicine C, Bispebjerg Hospital, Copenhagen, Denmark
}

Summary. Glomerular filtration rate, renal plasma flow, urinary excretion of $\beta$-2-microglobulin and albumin, heart rate and blood pressure were studied in eight young male insulin-dependent diabetics. Measurements were performed before and during insulin infusion at $2 \mathrm{mU} / \mathrm{kg} / \mathrm{min}$. No patient had discernible insulin antibodies. Two studies were performed at random in each patient. In series A blood glucose concentration was allowed to decline, while in series B it was maintained at a constant level. Ten 20 min clearance periods were performed, four before and six during insulin infusion. Results are given as mean \pm SEM of values from the first four (control) and last four (test) clearance periods. Blood glucose declined in series A experiments from $10.8 \pm 0.8$ $\mathrm{mmol} / 1$ in the control period to $5.8 \pm 0.5 \mathrm{mmol} / 1$ during the test period, but remained constant during experiment B $(9.8 \pm 1.1$ and $9.5 \pm 1.1 \mathrm{mmol} / \mathrm{l})$. Plasma insulin levels were comparable in the two series. Glomerular filtration rate fell from $141 \pm 7 \mathrm{ml} /$ $\min \times 1.73 \mathrm{~m}^{2}$ to $132 \pm 7 \mathrm{ml} / \mathrm{min} \times 1.73 \mathrm{~m}^{2}(\mathrm{p}<$ 0.01 ) in series A but did not change significantly during series B. Similarily renal plasma flow declined with declining glucose but remained constant when glucose was maintained at a constant level. In series A the magnitude of decrease in renal plasma flow was correlated with the magnitude of decrease in glomerular filtration rate $(\mathrm{r}=0.95, \mathrm{p}<0.001) . \beta$ 2 -microglobulin excretion decreased significantly ( $\mathrm{p}$ $<0.05$ ) in both series (A: $89 \pm 17$ to $60 \pm 13 \mathrm{ng} / \mathrm{min}$, B: $117 \pm 46$ to $62 \pm 17 \mathrm{ng} / \mathrm{min})$. Albumin excretion increased in five out of six patients with normal control values (not significant) in series $A$ and in four out of six in series B. No significant changes in heart rate or blood pressure were observed. Thus insulin infusion reduced renal plasma flow and glomerular filtration rate, but this effect could be completely abolished by keeping blood glucose constant. This suggests that it is not the lack of insulin but the associated hyperglycaemia which contributes to the elevated renal plasma flow and glomerular filtration rate in insulin-dependent diabetics.

Key words: Insulin-dependent diabetes, glomerular filtration rate, renal plasma flow, insulin infusion, hyperglycaemia, $\beta$-2-microglobulin, albumin

Recently, Mogensen et al. [1] have reported a decrease in glomerular filtration rate (GFR) $(-9 \%)$ and in renal plasma flow (RPF) $(-13 \%)$ in insulindependent diabetic patients (IDD) after IV injection of 7 to $8 \mathrm{U}$ of insulin. Plasma glucose decreased from $13.9 \mathrm{mmol} / 1$ to $6.5 \mathrm{mmol} / \mathrm{l}$. These findings suggest that the lack of insulin either per se or secondary to the change in blood glucose, may contribute to the elevated GFR and RPF demonstrated in short-term $\operatorname{IDD}[2,3]$.

We have tested these two hypotheses in IDD patients by measuring GFR, RPF and urinary excretion rates of albumin and $\beta$-2-microglobulin before and during IV insulin infusion resulting in plasma insulin levels within the postprandial range for normal man. The investigation was carried out on two occasions. In the first blood glucose was allowed to decline and in the second was maintained constant by infusion of glucose.

\section{Material and Methods}

\section{Patients}

Eight male IDD patients without detectable insulin antibodies [4] participated in the study after informed consent (Table 1). All were out-patients treated with two daily injections of highly purified porcine insulin (Retard Leo RI and Leo Neutral RI). 
Table 1. Pertinent clinical data of the eight male insulin-dependent diabetics studied

\begin{tabular}{lllccc}
\hline Subject & $\begin{array}{l}\text { Age } \\
\text { (years) }\end{array}$ & $\begin{array}{l}\text { Duration } \\
\text { of diabetes } \\
\text { (years) }\end{array}$ & $\begin{array}{l}\text { Ideal weight } \\
(\%)\end{array}$ & $\begin{array}{l}\text { Surface } \\
\text { area }\left(\mathbf{m}^{2}\right)\end{array}$ & $\begin{array}{l}\text { Insulin dose } \\
(\mathrm{U} / 24 \mathrm{~h} \cdot \mathrm{kg})\end{array}$ \\
\hline 1 & 23 & 11 & 92 & 1.92 & 0.65 \\
2 & 36 & 15 & 109 & 2.02 & 0.43 \\
3 & 32 & 1 & 90 & 2.00 & 0.35 \\
4 & 28 & 4 & 117 & 1.88 & 0.34 \\
5 & 38 & 8 & 100 & 1.97 & 0.39 \\
6 & 22 & 7 & 105 & 2.10 & 0.67 \\
7 & 32 & 0.2 & 89 & 1.95 & 0.27 \\
8 & 22 & 0.2 & $99 \pm 10$ & $1.97 \pm 0.07$ & 0.52 \\
\hline Mean \pm SD & $29 \pm 6$ & $6 \pm 5$ & & & $0.45 \pm 0.05$ \\
\hline
\end{tabular}
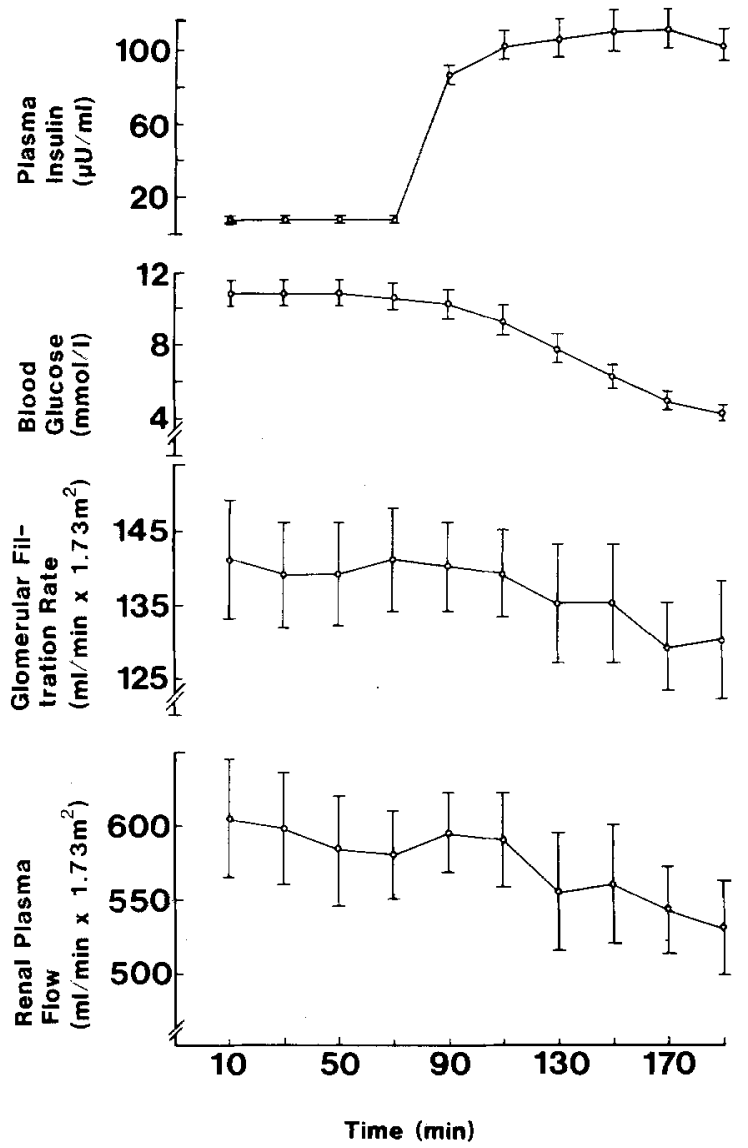

Fig. 1. Plasma-insulin $(n=6)$, blood-glucose, glomerular filtration rate and renal plasma flow in 8 insulin-dependent diabetics before and during IV insulin infusion (series A) (mean \pm SEM)

Patients 2, 5 and 6 had background retinopathy. No patients had persistent proteinuria, but patient 2 had intermittently Albustixpositive 24 hour urine specimens. All had normal serum creatinine. None of the patients received any drugs other than insulin. Ketone bodies were not present in their urine at the time of the investigations.

\begin{abstract}
Methods
GFR and RPF were measured using constant infusion techniques with ${ }^{125} \mathrm{I}$-iothalamate and ${ }^{131} \mathrm{I}$-hippuran respectively [2]. Thyroid uptake of radioactive iodide was blocked with potassium iodide $100 \mathrm{mg}$ for the two days prior to the study. Urinary concentrations of albumin and $\beta$-2-microglobulin (Phadebas, $\beta$-2-micro test) were measured by radioimmunoassay $[5,6]$. In patients $1-6$ and patient 8 plasma insulin concentrations were measured by radioimmunoassay [4] (measurements were unsuccessful in patient 7). In each clearance period haematocrit was determined in duplicate by $30 \mathrm{~min}$ centrifugation at $1500 \mathrm{~g}$, and total plasma protein concentration assessed with a T.S-meter (American Optical Company). Heart rate $(60 \mathrm{~s})$ and auscultatory blood pressure were measured at the beginning of each $20 \mathrm{~min}$ clearance period. Other measurements were performed in the middle of each clearance period.
\end{abstract}

\section{Procedure}

Experiments were performed in the morning following an overnight fast and were carried out in the supine position, the patients standing only to void urine. The last injection of insulin was taken $24 \mathrm{~h}$ prior to the studies. The patients were asked to drink $1 \mathrm{l}$ of tap water in the morning and were given $0.751 / \mathrm{h}$ during the experiments.

At $08.00 \mathrm{~h}$ the subjects were connected to a continuous blood glucose monitor (Biostator, Miles Company, Ulm) [7]. Measurement of clearance during four control periods was followed by six clearance periods during IV infusion of insulin. Each clearance period lasted $20 \mathrm{~min}$. Insulin (Insulin Leo Neutral RI) was diluted with $0.154 \mathrm{mmol} / \mathrm{l}$ saline and infused at $2 \mathrm{mU} / \mathrm{kg} / \mathrm{min}$, the infusion rate being $1 \mathrm{ml} / \mathrm{min}$.

Two experiments were carried out on each patient in random order with at least a 2 week interval. In one experimental series (A) the blood glucose was allowed to decline during insulin infusion, glucose being given only if the blood glucose fell below $3.5 \mathrm{mmol} / \mathrm{l}$, in which case an infusion of $50 \mathrm{~g} / 100 \mathrm{ml}$ glucose at $2 \mathrm{ml} / \mathrm{min}$ was started. Mean glucose infusion during these experiments was $12.5 \mathrm{ml} 50 \mathrm{~g} / 100 \mathrm{ml}$ glucose, range $0-45 \mathrm{ml}$. In the second series (B) blood glucose was kept at nearly constant levels by infusion of $20 \mathrm{~g} / 100 \mathrm{ml}$ glucose (mean $255 \mathrm{ml}$, range $200-300 \mathrm{ml}$ ), the infusion rate being operated manually but guided by the continuous blood glucose measurements of the Biostator. Except for the glucose infusion the procedures of the two experimental series were identical. 
Table 2. Renal function and heart rate during intravenous insulin infusion in eight IDD patients. (Series A). The control period consists of the first four $20 \mathrm{~min}$ clearance periods, and the insulin period the last four of the six insulin infusion clearance periods. Values are mean \pm SEM

\begin{tabular}{|c|c|c|c|c|c|c|c|c|c|c|}
\hline Subject & Period & $\begin{array}{l}\text { Plasma } \\
\text { insulin } \\
\mathrm{mU} / 1\end{array}$ & $\begin{array}{l}\text { Blood } \\
\text { glucose } \\
(\mathrm{mmol} / 1)\end{array}$ & $\begin{array}{l}\text { Glomerular } \\
\text { filtration } \\
\text { rate } \\
(\mathrm{ml} / \mathrm{min} \text {. } \\
\left.1.73 \mathrm{~m}^{2}\right)\end{array}$ & $\begin{array}{l}\text { Renal } \\
\text { plasma flow } \\
(\mathrm{ml} / \mathrm{min} \cdot \\
1.73 \mathrm{~m}^{2} \text { ) }\end{array}$ & $\begin{array}{l}\text { Filtration } \\
\text { fraction }\end{array}$ & $\begin{array}{l}\text { Urinary } \\
\text { albumin } \\
\text { excretion } \\
(\mu g / \mathrm{min})\end{array}$ & $\begin{array}{l}\text { Urinary } \\
\beta \text {-2-micro- } \\
\text { globulin } \\
\text { excretion } \\
(\mathrm{ng} / \mathrm{min})\end{array}$ & $\begin{array}{l}\text { Unine } \\
\text { flow } \\
(\mathrm{ml} / \mathrm{min})\end{array}$ & $\begin{array}{l}\text { Heart } \\
\text { rate } / \mathrm{min}\end{array}$ \\
\hline 1 & $\begin{array}{l}\text { Control } \\
\text { Insulin }\end{array}$ & $\begin{array}{r}5 \pm 0 \\
94 \pm 5\end{array}$ & $\begin{array}{r}13.1 \pm 0.2 \\
6.2 \pm 1.1\end{array}$ & $\begin{array}{l}137 \pm 3 \\
130 \pm 3\end{array}$ & $\begin{array}{l}552 \pm 3 \\
515 \pm 8\end{array}$ & $\begin{array}{l}0.25 \\
0.25\end{array}$ & $\begin{array}{l}3 \pm 1 \\
5 \pm 1\end{array}$ & $\begin{array}{l}72 \pm 1 \\
52 \pm 1\end{array}$ & $\begin{array}{l}17.5 \pm 0.2 \\
14.6 \pm 0.8\end{array}$ & $\begin{array}{l}65 \pm 1 \\
63 \pm 1\end{array}$ \\
\hline 2 & $\begin{array}{l}\text { Control } \\
\text { Insulin }\end{array}$ & $\begin{array}{r}4 \pm 0 \\
103 \pm 1\end{array}$ & $\begin{array}{r}11.2 \pm 0.1 \\
8.2 \pm 0.5\end{array}$ & $\begin{array}{r}110 \pm 1 \\
97 \pm 2\end{array}$ & $\begin{array}{l}489 \pm 9 \\
430 \pm 8\end{array}$ & $\begin{array}{l}0.23 \\
0.23\end{array}$ & $\begin{array}{l}246 \pm 8 \\
285 \pm 11\end{array}$ & $\begin{array}{r}140 \pm 4 \\
53 \pm 3\end{array}$ & $\begin{array}{l}4.4 \pm 0.1 \\
1.1 \pm 0.2\end{array}$ & $\begin{array}{l}80 \pm 1 \\
88 \pm 1\end{array}$ \\
\hline 3 & $\begin{array}{l}\text { Control } \\
\text { Insulin }\end{array}$ & $\begin{array}{l}14 \pm 0 \\
98 \pm 8\end{array}$ & $\begin{array}{l}7.8 \pm 0.0 \\
3.9 \pm 0.1\end{array}$ & $\begin{array}{l}121 \pm 1 \\
106 \pm 1\end{array}$ & $\begin{array}{l}509 \pm 11 \\
429 \pm 1\end{array}$ & $\begin{array}{l}0.24 \\
0.25\end{array}$ & $\begin{array}{l}4 \pm 1 \\
5 \pm 1\end{array}$ & $\begin{array}{l}24 \pm 1 \\
32 \pm 5\end{array}$ & $\begin{array}{l}13.5 \pm 0.2 \\
15.8 \pm 0.4\end{array}$ & $\begin{array}{l}77 \pm 1 \\
74 \pm 1\end{array}$ \\
\hline 4 & $\begin{array}{l}\text { Control } \\
\text { Insulin }\end{array}$ & $\begin{array}{r}10 \pm 0 \\
108 \pm 3\end{array}$ & $\begin{array}{r}11.0 \pm 0.1 \\
5.6 \pm 1.1\end{array}$ & $\begin{array}{l}162 \pm 1 \\
145 \pm 6\end{array}$ & $\begin{array}{l}762 \pm 30 \\
687 \pm 24\end{array}$ & $\begin{array}{l}0.21 \\
0.21\end{array}$ & $\begin{array}{r}4 \pm 1 \\
14 \pm 3\end{array}$ & $\begin{array}{r}117 \pm 9 \\
67 \pm 5\end{array}$ & $\begin{array}{l}13.2 \pm 0.1 \\
14.6 \pm 0.1\end{array}$ & $\begin{array}{l}58 \pm 0 \\
60 \pm 0\end{array}$ \\
\hline 5 & $\begin{array}{l}\text { Control } \\
\text { Insulin }\end{array}$ & $\begin{array}{r}4 \pm 0 \\
98 \pm 8\end{array}$ & $\begin{array}{r}11.1 \pm 0.0 \\
5.8 \pm 1.0\end{array}$ & $\begin{array}{l}159 \pm 1 \\
155 \pm 2\end{array}$ & $\begin{array}{l}685 \pm 18 \\
677 \pm 5\end{array}$ & $\begin{array}{l}0.23 \\
0.23\end{array}$ & $\begin{array}{l}97 \pm 2 \\
63 \pm 2\end{array}$ & $\begin{array}{l}28 \pm 2 \\
45 \pm 5\end{array}$ & $\begin{array}{l}16.1 \pm 0.3 \\
18.1 \pm 0.4\end{array}$ & $\begin{array}{l}66 \pm 0 \\
68 \pm 0\end{array}$ \\
\hline 6 & $\begin{array}{l}\text { Control } \\
\text { Insulin }\end{array}$ & $\begin{array}{r}10 \pm 0 \\
155 \pm 5\end{array}$ & $\begin{array}{r}13.7 \pm 0.0 \\
6.8 \pm 1.3\end{array}$ & $\begin{array}{l}131 \pm 1 \\
130 \pm 3\end{array}$ & $\begin{array}{l}514 \pm 12 \\
489 \pm 7\end{array}$ & $\begin{array}{l}0.26 \\
0.27\end{array}$ & $\begin{array}{l}14 \pm 2 \\
11 \pm 1\end{array}$ & $\begin{array}{l}78 \pm 8 \\
53 \pm 11\end{array}$ & $\begin{array}{l}16.6 \pm 0.1 \\
15.7 \pm 0.3\end{array}$ & $\begin{array}{l}57 \pm 1 \\
59 \pm 1\end{array}$ \\
\hline 7 & $\begin{array}{l}\text { Control } \\
\text { Insulin }\end{array}$ & a & $\begin{array}{l}7.9 \pm 0.1 \\
3.8 \pm 0.1\end{array}$ & $\begin{array}{l}160 \pm 2 \\
145 \pm 3\end{array}$ & $\begin{array}{l}606 \pm 7 \\
537 \pm 15\end{array}$ & $\begin{array}{l}0.26 \\
0.27\end{array}$ & $\begin{array}{l}12 \pm 2 \\
23 \pm 2\end{array}$ & $\begin{array}{l}92 \pm 11 \\
33 \pm 3\end{array}$ & $\begin{array}{l}10.2 \pm 0.3 \\
12.6 \pm 0.5\end{array}$ & $\begin{array}{l}55 \pm 1 \\
62 \pm 1\end{array}$ \\
\hline 8 & $\begin{array}{l}\text { Control } \\
\text { Insulin }\end{array}$ & $\begin{array}{r}6 \pm 0 \\
>63^{b}\end{array}$ & $\begin{array}{r}10.3 \pm 0.2 \\
6.1 \pm 0.8\end{array}$ & $\begin{array}{l}145 \pm 2 \\
144 \pm 2\end{array}$ & $\begin{array}{l}613 \pm 22 \\
608 \pm 18\end{array}$ & $\begin{array}{l}0.24 \\
0.24\end{array}$ & $\begin{array}{r}5 \pm 1 \\
24 \pm 3\end{array}$ & $\begin{array}{l}158 \pm 15 \\
143 \pm 4\end{array}$ & $\begin{array}{l}19.4 \pm 0.3 \\
24.3 \pm 0.3\end{array}$ & $\begin{array}{l}64 \pm 1 \\
64 \pm 0\end{array}$ \\
\hline $\begin{array}{l}\text { Mean } \\
( \pm \text { SEM })\end{array}$ & $\begin{array}{l}\text { Control } \\
\text { Insulin }\end{array}$ & $\begin{array}{r}7 \pm 2 \\
109 \pm 9 \\
p<0.01\end{array}$ & $\begin{array}{c}10.8 \pm 0.8 \\
5.8 \pm 0.5 \\
p<0.01\end{array}$ & $\begin{array}{l}141 \pm 7 \\
132 \pm 7 \\
p<0.01\end{array}$ & $\begin{array}{l}591 \pm 34 \\
547 \pm 36 \\
p<0.01\end{array}$ & $\begin{array}{l}0.24 \pm 0.01 \\
0.24 \pm 0.01\end{array}$ & $\begin{array}{l}48 \pm 30 \\
54 \pm 34\end{array}$ & $\begin{array}{c}89 \pm 17 \\
60 \pm 13 \\
p<0.05\end{array}$ & $\begin{array}{l}13.9 \pm 1.7 \\
14.6 \pm 2.3\end{array}$ & $\begin{array}{l}65 \pm 3 \\
67 \pm 3\end{array}$ \\
\hline
\end{tabular}

a Not measured

b Not measured on diluted sample

\section{Control Experiments}

The effect of sustained water diuresis on GFR and RPF was evaluated in three male IDD patients (mean age 29 years, range 26-31 years, mean duration of diabetes 11 years range 4-14 years) using the same procedure for clearance measurements as described above.

\section{Statistics}

The Wilcoxon matched-pairs test was used for comparison of mean values from the first four and the last four clearance periods, thus excluding the transitional clearance periods (5 and 6).

All values are corrected to $1.73 \mathrm{~m}^{2}$ body surface area. Results are stated as mean \pm SEM.

\section{Results}

During insulin infusion plasma levels of insulin increased from $7 \mathrm{mU} / 1$ to $109 \mathrm{mU} / 1$ in the first experimental series (A) (Table 2), with similar values during the other series (B) (Table 3). Blood glucose concentration declined during the first experiment
(A) from $10.8 \pm 0.8$ to $5.8 \pm 0.5 \mathrm{mmol} / \mathrm{l}$, while no significant change took place in the second series (B) $(9.8 \pm 1.1$ to $9.5 \pm 1.1 \mathrm{mmol} / \mathrm{l})$ (Tables 2 and 3 ).

Mean values of GFR, RPF, blood glucose and plasma insulin from each twenty minute clearance period in the experimental series with declining blood glucose (A) are shown in Figure 1.

Small but significant falls in GFR $(141 \pm 7$ to $\left.132 \pm 7 \mathrm{ml} / \mathrm{min} \cdot 1.73 \mathrm{~m}^{2}, \mathrm{p}<0.01\right)$ and in $\mathrm{RPF}$ $\left(591 \pm 34\right.$ to $547 \pm 36 \mathrm{ml} / \mathrm{min} \cdot 1.73 \mathrm{~m}^{2}, \mathrm{p}<0.01$ ), were observed in series A (Table 2). By contrast no changes in GFR or RPF were seen when blood glucose was kept at almost constant levels (B) (Table 3 ). Filtration fraction remained unchanged in both experimental series.

A significant correlation between changes in GFR and RPF was found $(\mathrm{r}=0.95, \mathrm{p}<0.001)$ (Fig. 2).

Patients 2 and 5 had elevated excretion rates of albumin in the control periods. Albumin excretion increased in five of the remaining six patients in series $A$ (not significant) and in four patients in series $B$ (not significant) (Tables 2 and 3). $\beta$-2-microglobulin 
Table 3. Renal function and heart rate during intravenous insulin infusion in eight IDD patients. Blood glucose kept constant. (Series B) The control and insulin periods were as for Table 2. Values are mean \pm SEM

\begin{tabular}{|c|c|c|c|c|c|c|c|c|c|c|}
\hline Subject & Period & $\begin{array}{l}\text { Plasma } \\
\text { insulin } \\
(\mu \mathrm{U} / \mathrm{ml})\end{array}$ & $\begin{array}{l}\text { Blood } \\
\text { glucose } \\
(\mathrm{mmol} / \mathrm{l})\end{array}$ & $\begin{array}{l}\text { Glomerular } \\
\text { filtration } \\
\text { rate } \\
(\mathrm{ml} / \mathrm{min} \text {. } \\
\left.1.73 \mathrm{~m}^{2}\right)\end{array}$ & $\begin{array}{l}\text { Renal } \\
\text { plasma flow } \\
(\mathrm{ml} / \mathrm{min} \cdot \\
\left.1.73 \mathrm{~m}^{2}\right)\end{array}$ & $\begin{array}{l}\text { Filtration } \\
\text { fraction }\end{array}$ & $\begin{array}{l}\text { Urináry } \\
\text { albumin } \\
\text { excretion } \\
\text { ( } \mu \mathrm{g} / \mathrm{min})\end{array}$ & $\begin{array}{l}\text { Urinary } \\
\beta \text {-2-micro- } \\
\text { globulin } \\
\text { excretion } \\
(\mathrm{ng} / \mathrm{min})\end{array}$ & $\begin{array}{l}\text { Urine } \\
\text { flow } \\
(\mathrm{ml} / \mathrm{min})\end{array}$ & $\begin{array}{l}\text { Heart } \\
\text { rate/min }\end{array}$ \\
\hline 1 & $\begin{array}{l}\text { Control } \\
\text { Insulin }\end{array}$ & $\begin{array}{r}4 \pm 0 \\
105 \pm 0\end{array}$ & $\begin{array}{l}9.0 \pm 0.1 \\
8.6 \pm 0.3\end{array}$ & $\begin{array}{l}134 \pm 1 \\
136 \pm 1\end{array}$ & $\begin{array}{l}513 \pm 4 \\
499 \pm 11\end{array}$ & $\begin{array}{l}0.26 \\
0.27\end{array}$ & $\begin{array}{l}4 \pm 1 \\
5 \pm 1\end{array}$ & $\begin{array}{l}73 \pm 1 \\
44 \pm 1\end{array}$ & $\begin{array}{l}11.0 \pm 0.2 \\
11.7 \pm 1.0\end{array}$ & $\begin{array}{l}58 \pm 1 \\
60 \pm 0\end{array}$ \\
\hline 2 & $\begin{array}{l}\text { Control } \\
\text { Insulin }\end{array}$ & $\begin{array}{r}4 \pm 0 \\
99 \pm 5\end{array}$ & $\begin{array}{l}13.4 \pm 0.0 \\
12.6 \pm 0.1\end{array}$ & $\begin{array}{l}102 \pm 1 \\
109 \pm 2\end{array}$ & $\begin{array}{l}484 \pm 7 \\
465 \pm 7\end{array}$ & $\begin{array}{l}0.21 \\
0.23\end{array}$ & $\begin{array}{l}337 \pm 8 \\
368 \pm 9\end{array}$ & $\begin{array}{l}\text { a } \\
\text { a }\end{array}$ & $\begin{array}{r}9.8 \pm 0.2 \\
13.7 \pm 0.2\end{array}$ & $\begin{array}{l}71 \pm 1 \\
85 \pm 1\end{array}$ \\
\hline 3 & $\begin{array}{l}\text { Control } \\
\text { Insulin }\end{array}$ & $\begin{array}{r}9 \pm 1 \\
125 \pm 2\end{array}$ & $\begin{array}{l}7.8 \pm 0.0 \\
7.8 \pm 0.1\end{array}$ & $\begin{array}{l}150 \pm 2 \\
147 \pm 3\end{array}$ & $\begin{array}{l}662 \pm 15 \\
625 \pm 31\end{array}$ & $\begin{array}{l}0.23 \\
0.24\end{array}$ & $\begin{array}{l}6 \pm 1 \\
4 \pm 1\end{array}$ & $\begin{array}{l}33 \pm 1 \\
42 \pm 5\end{array}$ & $\begin{array}{l}16.1 \pm 0.1 \\
17.2 \pm 0.3\end{array}$ & $\begin{array}{l}69 \pm 2 \\
65 \pm 2\end{array}$ \\
\hline 4 & $\begin{array}{l}\text { Control } \\
\text { Insulin }\end{array}$ & $\begin{array}{r}6 \pm 0 \\
81 \pm 6\end{array}$ & $\begin{array}{l}5.0 \pm 0.0 \\
4.7 \pm 0.0\end{array}$ & $\begin{array}{l}135 \pm 1 \\
127 \pm 1\end{array}$ & $\begin{array}{l}546 \pm 10 \\
573 \pm 6\end{array}$ & $\begin{array}{l}0.25 \\
0.22\end{array}$ & $\begin{array}{l}7 \pm 1 \\
6 \pm 1\end{array}$ & $\begin{array}{l}30 \pm 4 \\
24 \pm 3\end{array}$ & $\begin{array}{r}9.8 \pm 0.1 \\
11.2 \pm 0.1\end{array}$ & $\begin{array}{l}46 \pm 1 \\
50 \pm 1\end{array}$ \\
\hline 5 & $\begin{array}{l}\text { Control } \\
\text { Insulin }\end{array}$ & $\begin{array}{r}8 \pm 1 \\
107 \pm 6\end{array}$ & $\begin{array}{l}11.4 \pm 0.1 \\
10.9 \pm 0.2\end{array}$ & $\begin{array}{l}149 \pm 1 \\
154 \pm 3\end{array}$ & $\begin{array}{l}619 \pm 11 \\
629 \pm 12\end{array}$ & $\begin{array}{l}0.24 \\
0.25\end{array}$ & $\begin{array}{l}91 \pm 2 \\
76 \pm 1\end{array}$ & $\begin{array}{l}44 \pm 4 \\
36 \pm 6\end{array}$ & $\begin{array}{l}11.5 \pm 0.8 \\
16.3 \pm 0.7\end{array}$ & $\begin{array}{l}60 \pm 1 \\
63 \pm 0\end{array}$ \\
\hline 6 & $\begin{array}{l}\text { Control } \\
\text { Insulin }\end{array}$ & $\begin{array}{r}6 \pm 0 \\
141 \pm 5\end{array}$ & $\begin{array}{l}13.7 \pm 0.1 \\
14.0 \pm 0.1\end{array}$ & $\begin{array}{l}133 \pm 1 \\
141 \pm 1\end{array}$ & $\begin{array}{l}580 \pm 11 \\
578 \pm 5\end{array}$ & $\begin{array}{l}0.23 \\
0.24\end{array}$ & $\begin{array}{l}6 \pm 1 \\
8 \pm 1\end{array}$ & $\begin{array}{r}133 \pm 8 \\
52 \pm 1\end{array}$ & $\begin{array}{r}8.6 \pm 0.9 \\
14.5 \pm 0.6\end{array}$ & $\begin{array}{l}61 \pm 0 \\
62 \pm 0\end{array}$ \\
\hline 7 & $\begin{array}{l}\text { Control } \\
\text { Insulin }\end{array}$ & $\begin{array}{l}\text { a } \\
\text { a }\end{array}$ & $\begin{array}{l}7.2 \pm 0.1 \\
6.0 \pm 0.1\end{array}$ & $\begin{array}{l}174 \pm 1 \\
174 \pm 1\end{array}$ & $\begin{array}{l}596 \pm 3 \\
632 \pm 9\end{array}$ & $\begin{array}{l}0.29 \\
0.28\end{array}$ & $\begin{array}{l}12 \pm 2 \\
12 \pm 3\end{array}$ & $\begin{array}{l}50 \pm 13 \\
28 \pm 2\end{array}$ & $\begin{array}{r}8.1 \pm 0.4 \\
11.7 \pm 0.3\end{array}$ & $\begin{array}{l}54 \pm 1 \\
56 \pm 1\end{array}$ \\
\hline 8 & $\begin{array}{l}\text { Control } \\
\text { Insulin }\end{array}$ & $\begin{array}{r}6 \pm 0 \\
>63^{b}\end{array}$ & $\begin{array}{l}10.9 \pm 0.1 \\
11.3 \pm 0.2\end{array}$ & $\begin{array}{l}145 \pm 1 \\
143 \pm 1\end{array}$ & $\begin{array}{l}601 \pm 7 \\
553 \pm 8\end{array}$ & $\begin{array}{l}0.24 \\
0.26\end{array}$ & $\begin{array}{l}15 \pm 2 \\
43 \pm 5\end{array}$ & $\begin{array}{l}420 \pm 14 \\
159 \pm 7\end{array}$ & $\begin{array}{l}19.1 \pm 0.1 \\
21.0 \pm 0.1\end{array}$ & $\begin{array}{l}61 \pm 1 \\
67 \pm 1\end{array}$ \\
\hline $\begin{array}{l}\text { Mean } \\
( \pm S E M)\end{array}$ & $\begin{array}{l}\text { Control } \\
\text { Insulin }\end{array}$ & $\begin{array}{r}6 \pm 1 \\
110 \pm 9 \\
p<0.01\end{array}$ & $\begin{array}{l}9.8 \pm 1.1 \\
9.5 \pm 1.1\end{array}$ & $\begin{array}{l}140 \pm 7 \\
141 \pm 7\end{array}$ & $\begin{array}{l}575 \pm 21 \\
569 \pm 22\end{array}$ & $\begin{array}{l}0.24 \pm 0.01 \\
0.25 \pm 0.01\end{array}$ & $\begin{array}{l}60 \pm 41 \\
65 \pm 44\end{array}$ & $\begin{array}{c}117 \pm 46 \\
62 \pm 17 \\
p<0.05\end{array}$ & $\begin{array}{l}11.8 \pm 1.4 \\
14.7 \pm 1.2 \\
\mathrm{p}<0.01\end{array}$ & $\begin{array}{l}60 \pm 3 \\
64 \pm 4\end{array}$ \\
\hline
\end{tabular}

a Not measured

b Not measured on diluted sample

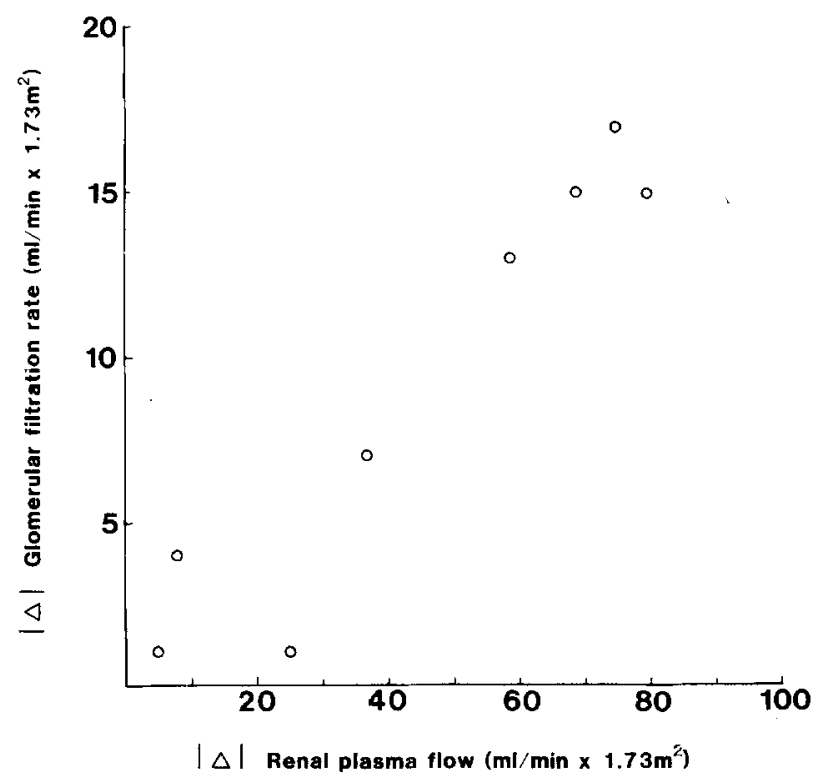

Fig. 2. Comparison of changes (decline) in renal plasma flow and glomerular filtration rate in 8 insulin-dependent diabetics during IV insulin infusion (series A) excretion was reduced following insulin infusion in both series (Tables 2 and 3 ). In the first series (A) no significant changes in urinary output were associated with insulin infusion (Table 2), while a significant increase from $11.8 \pm 1.4$ to $14.7 \pm 1.2 \mathrm{ml} / \mathrm{min}(\mathrm{p}<$ 0.01 ) was seen during insulin infusion with constant blood glucose levels (B) (Table 3).

Haematocrit was unchanged in series A (45.9 \pm 1.1 versus $45.3 \pm 1.1 \%$ ) while a significant reduction was observed in the other series (B) $46.3 \pm 0.7$ versus $45.0 \pm 0.6 \%(\mathrm{p}<0.01)$. Corresponding values for plasma protein concentrations were: A: $69 \pm 4$ versus $68 \pm 4 \mathrm{~g} / 1(\mathrm{p}<0.05)$ and $\mathrm{B}: 69 \pm 6 \mathrm{~g} / \mathrm{l}$ versus $65 \pm 4 \mathrm{~g} / 1(\mathrm{p}<0.01)$

No significant changes in blood pressure or heart rate took place in either of the series, neither in the test clearance periods nor in the transitional clearance periods compared to the control periods.

Sustained water diuresis with clearance measurements for 240 minutes in three IDD patients revealed no systematic alterations in GFR or RPF (Table 4). 
Table 4. Effect of sustained water diuresis on glomerular filtration rate and renal plasma flow in three male insulin-dependent diabetics

\begin{tabular}{|c|c|c|c|c|c|}
\hline Subject & $\begin{array}{l}\text { Period } \\
\text { min }\end{array}$ & $\begin{array}{l}\text { Glomerular } \\
\text { filtration rate } \\
\left(\mathrm{ml} / \mathrm{min} \cdot 1.73 \mathrm{~m}^{2}\right)\end{array}$ & $\begin{array}{l}\text { Renal } \\
\text { plasma flow } \\
\left(\mathrm{ml} / \mathrm{min} \cdot 1.73 \mathrm{~m}^{2}\right)\end{array}$ & $\begin{array}{l}\text { Filtration } \\
\text { fraction }\end{array}$ & $\begin{array}{l}\text { Urine flow } \\
(\mathrm{ml} / \mathrm{min})\end{array}$ \\
\hline I & $\begin{array}{r}0-120^{\mathrm{a}} \\
121-240^{\mathrm{a}}\end{array}$ & $\begin{array}{l}146 \pm 2 \\
142 \pm 2\end{array}$ & $\begin{array}{l}582 \pm 6 \\
564 \pm 5\end{array}$ & $\begin{array}{l}0.25 \\
0.25\end{array}$ & $\begin{array}{l}10.6 \pm 0.3 \\
12.6 \pm 0.2\end{array}$ \\
\hline II & $\begin{array}{r}0-120 \\
121-240\end{array}$ & $\begin{array}{l}157 \pm 2 \\
153 \pm 3\end{array}$ & $\begin{array}{l}681 \pm 5 \\
666 \pm 6\end{array}$ & $\begin{array}{l}0.23 \\
0.23\end{array}$ & $\begin{array}{l}13.7 \pm 0.4 \\
13.0 \pm 0.3\end{array}$ \\
\hline III & $\begin{array}{c}0-120 \\
121 \pm 240\end{array}$ & $\begin{array}{l}128 \pm 2 \\
132 \pm 2\end{array}$ & $\begin{array}{l}427 \pm 4 \\
429 \pm 5\end{array}$ & $\begin{array}{l}0.30 \\
0.30\end{array}$ & $\begin{array}{l}12.4 \pm 0.3 \\
12.9 \pm 0.2\end{array}$ \\
\hline
\end{tabular}

a Values given as mean of six 20 min clearance periods $( \pm$ SEM)

\section{Discussion}

In the present paper it is confirmed that IV insulin infusion in IDD patients results in decreases in RPF and GFR. However, it is further demonstrated that this effect can be completely abolished when blood glucose is kept constant by concomitant glucose infusion.

A decline in RPF $(-13 \%)$ and in GFR $(-9 \%)$ was previously found in IDD patients, after an intravenous bolus injection of 7 to $8 \mathrm{U}$ of insulin [1]. Plasma insulin concentration was not recorded, but must have been outside the physiological range, probably more than $1000 \mathrm{mU} / 1$ initially. In the present study we evaluated the effect on kidney function in IDD patients of an IV insulin infusion resulting in plasma insulin levels $(109 \mathrm{mU} / 1)$ within the postprandial range of normal man [8]. We found a gradual reduction in RPF $(-7 \%)$ and in GFR $(-6 \%)$. This effect was present in all eight IDD patients studied (Table 2).

Mogensen et al. [1] suggested that the effect of insulin on renal haemodynamics was most likely due to increased sympathetic nervous activity, but a direct effect of insulin or of glucose on renal function could not be excluded. The suggestion of increased sympathetic nervous activity as the dominant mechanism involved was based upon prior reports of enhanced heart rate and increased plasma noradrenalin concentration in IDD patients after an intravenous bolus injection of 6 to $20 \mathrm{U}$ of insulin [9, $10,11]$. Heart rate did not however increase in our study (A) with plasma insulin levels within the physiological range.

It is well documented that a close relationship exists between RPF and GFR, and that RPF is one of the major determinants of GFR, as reviewed by Brenner and Humes [12]. Our study also revealed a close relationship between these two variables (Fig. 2), although it has to be remembered that errors in urine collection will affect RPF and GFR equally and thus induce a tendency to a false correlation. However, the influence of such errors is reduced by using the mean of results from four clearance periods. Arterial blood pressure and filtration fraction remained unchanged during the insulin infusion, suggesting that the decrease in RPF is due to a parallel increase in the hydraulic resistance of the preglomerular and the postglomerular arterioles. Our results suggest that the decrease in RPF is the dominant cause of the GFR reduction induced by insulin infusion.

To evaluate if the demonstrated decrease in RPF and GFR was due to insulin per se, we carried out exactly the same investigation in the same eight IDD patients as mentioned above, but kept the blood glucose constant. When blood glucose was kept constant insulin infusion resulting in plasma insulin concentrations within the physiological range (mean $110 \mathrm{mU} / \mathrm{l}$ ) did not affect RPF or GFR. Similar results have been obtained in normal man when blood glucose was kept constant during insulin infusion at the same rate as presently used [13] and after an intravenous bolus injection of $20 \mathrm{U}$ of insulin [14]. This lack of insulin effect on kidney function may either be due to constant blood glucose or plasma volume expansion induced by the glucose infusion $(255 \mathrm{ml}, 20 \mathrm{~g} / 100 \mathrm{ml}$ glucose in our study). This infusion decreased the haemetaocrit from 46.3 to 45.0 . We have, however, shown that RPF and GFR remain constant in normal man during intravenous infusion of $0.154 \mathrm{mmol} / 1$ saline at $600 \mathrm{ml} / \mathrm{h}$ with a decrease in haematocrit from 45.3 to 43.3 [15]. In the same study [15] glucose infusion in normal man with an increase in blood glucose from $4.7 \mathrm{mmol} / 1$ to $10.9 \mathrm{mmol} / 1$ resulted in an increase in GFR of $6 \%$. Furthermore it has recently been reported that glucose infusion resulting in a significant rise in blood glucose levels can abolish the effect on RPF and GFR of insulin injection in IDD [16]. 
Mogensen et al. [1] found an increase in urinary albumin excretion after intravenous bolus injection of 7 to $8 \mathrm{U}$ of insulin 5 IDD patients, whereas Viberti et al. [17] found no significant changes in urinary albumin excretion after intravenous bolus injection of 8 to $10 \mathrm{U}$ of insulin in 5 IDD patients. In order to compare the present urinary albumin excretion findings with the two above mentioned studies, patients 2 and 5 were omitted from calculations since both had a slightly elevated albumin excretion rate in the control state. Urinary albumin excretion was found to increase in five of the remaining six patients $(0.1>p$ $>0.05$ ) in series $A$, and in four of six (not significant) in series B.

In accordance with Mogensen et al. [1] we found a significant reduction in $\beta$-2-microglobulin excretion rate. Since this effect was also present in the studies where blood glucose was kept constant, this suggests that insulin enhances tubular protein reabsorption.

The results of the present study lead us to conclude that insulin per se does not induce alterations in RPF and GFR, since this effect is dependent of the concomitant blood glucose changes. Even more important, the above mentioned findings suggest that it is not the lack of insulin but the associated hyperglycaemia which contributes to the elevated GFR and RPF in IDD patients.

\section{References}

1. Mogensen CE, Christensen NJ, Gundersen HJG (1978) The acute effect of insulin on renal hemodynamics and protein excretion in diabetics. Diabetologia 15: 153-157

2. Mogensen CE (1971) Glomerular filtration rate and renal plasma flow in short-term and long-term juvenile diabetics. Scand J Clin Lab Invest 28: 91-100

3. Christiansen J Sandahl, Parving H-H, Gammelgaard J, Holm H-H, Svendsen P Aa (1979) Kidney function and kidney size in newly diagnosed insulin-dependent diabetics before and during initial insulin therapy. Excerpta Med 481: 202

4. Heding L (1972) Determination of total serum insulin (IRI) in insulin treated diabetic patients. Diabetologia 8: 260-266

5. Miles DW, Mogensen CE, Gundersen HJG (1970) Radioimmunoassay for urinary albumin using a single antibody. Scand $\mathrm{J}$ Clin Lab Invest 26: 5-11
6. Evrin PE, Petersen PA, Wide L, Berggård J (1971) Radioimmunoassay of $\beta$-2-microglobulin in human biological fluids. Scand J Clin Lab Invest 28: 439-444

7. Pfeiffer EF, Thum $\mathrm{Ch}$, Clemens AH (1974) The artificial betacell - A continuous control of blood sugar by external regulation of insulin infusion (Glucose controlled insulin infusion system). Horm Metab Res 487: 339-342

8. Deckert T, Bojsen J, Christiansen J Sandahl, Kølendorf K, Svendsen P Aa, Andersen AR (in press) $24 \mathrm{~h}$ blood glucose profiles in insulin dependent diabetics treated by intravenous insulin infusion systems. A comparison of closed- and open loop-systems. Acta Med Scand

9. Page M McB, Smith RBW, Watkins PJ (1976) Cardiovascular effects of insulin. Br Med J I: $430-432$

10. Gundersen HJG, Christensen NJ (1977) Intravenous insulin causing loss of intravascular water and albumin and increased nervous activity in diabetics. Diabetes 26: 551-557

11. Parving H-H, Noer I, Deckert T, Lassen NA (1979) Intravenous insulin has no effect on transcapillary escape rate of albumin and on plasma volume in short-term juvenile diabetics. Diabetes 28: 282-286

12. Brenner B, Humes HD (1977) Mechanisms of glomerular ultrafiltration. N Engl J Med 297: 148-154

13. DeFronzo RA, Cooke CR, Andres R, Faloona GR, Davis PJ (1975) The effect of insulin on renal handling of sodium, potassium, calcium and phosphate in man. J Clin Invest 55: 845-855

14. Miller JH (1953) Effect of insulin on maximal rate of renal tubular uptake of glucose in non-diabetic humans. Proc Soc Exp Biol Med 84: 322-324

15. Parving H-H, Christiansen J Sandahl, Frandsen M (1980) Elevated glomerular filtration rate during moderate hyperglycemia in normal man. Acta Endocrinol (Kbh) 94 [Suppl 237]: 68

16. Mogensen CE, Christensen NJ, Gundersen HJG (1980) The acute effect of insulin on heart rate, blood pressure, plasma noradrenaline and urinary albumin excretion. Diabetologia 18: $453-457$

17. Viberti GC, Haycock GB, Pickup JC, Jarrett RJ, Keen H (1980) Early functional and morphologic vascular renal consequences of the diabetic state. Diabetologia 18: 173-175

Received: June 23, 1980, and in revised form: October 16, 1980

\author{
J. Sandahl Christiansen, M. D. \\ Steno Memorial Hospital \\ DK-2820 Gentofte \\ Denmark
}

